University of South Carolina

Scholar Commons

$5-17-2010$

\title{
Laboratory Bounds on Electron Lorentz Violation
}

Brett David Altschul

altschul@mailbox.sc.edu

Follow this and additional works at: https://scholarcommons.sc.edu/phys_facpub

Part of the Physics Commons

\section{Publication Info \\ Published in Physical Review D, Volume 82, Issue 1, 2010. (C2010 American Physical Society}

This Article is brought to you by the Physics and Astronomy, Department of at Scholar Commons. It has been accepted for inclusion in Faculty Publications by an authorized administrator of Scholar Commons. For more information, please contact digres@mailbox.sc.edu. 


\title{
Laboratory Bounds on Electron Lorentz Violation
}

\author{
Brett Altschul1 \\ Department of Physics and Astronomy \\ University of South Carolina \\ Columbia, SC 29208
}

\begin{abstract}
Violations of Lorentz boost symmetry in the electron and photon sectors can be constrained by studying several different high-energy phenomenon. Although they may not lead to the strongest bounds numerically, measurements made in terrestrial laboratories produce the most reliable results. Laboratory bounds can be based on observations of synchrotron radiation, as well as the observed absences of vacuum Cerenkov radiation $\left(e^{ \pm} \rightarrow e^{ \pm}+\gamma\right)$ and photon decay $\left(\gamma \rightarrow e^{+}+e^{-}\right)$. Using measurements of synchrotron energy losses at LEP and the survival of $\mathrm{TeV}$ photons, we place new bounds on the three electron Lorentz violation coefficients $c_{(T J)}$, at the $3 \times 10^{-13}$ to $6 \times 10^{-15}$ levels.
\end{abstract}

\footnotetext{
${ }^{1}$ baltschu@physics.sc.edu
} 


\section{Introduction}

Recent years have seen a surge of interest in the possibility that apparently fundamental symmetries, such as Lorentz and CPT invariances, may not be exact in nature. The scope and precision of experimental tests of such symmetry violations have expanded tremendously, but no compelling experimental evidence for violation of Lorentz invariance or CPT has been seen. Nonetheless, this remains a very active field of research. One reason for this is that the payoff from any discovery would be amazingly high; such symmetry violations (or other similar effects that could not occur within the known contexts of the standard model and general relativity) would be immediate evidence for new physics of a completely unheralded character. For example, violations of Lorentz symmetry or CPT could be tied to the structure of quantum gravity and would provide crucial clues as to what other novel effects we should look for.

All possible forms of Lorentz and CPT violations consistent with quantum theory as we understand it can be parameterized through an effective field theory called the standard model extension (SME). The SME contains Lorentz- and CPT-violating corrections to the standard model [1, 2]. (The framework can also be expanded to cover gravity [3].) Both the renormalizability [4, 5] and stability [6] of the SME have been studied. The SME is now the standard framework for parameterizing the results of experimental Lorentz and CPT tests. With the systematic description made possible by the SME, experimentalists can study a wider range of symmetry-breaking phenomena; most possible forms of Lorentz and CPT violation were overlooked in earlier, purely phenomenalistic analyses.

Recent searches for Lorentz violation have included studies of matter-antimatter asymmetries for trapped charged particles [7, 8, 9] and bound state systems [10, 11], measurements of muon properties [12, 13], analyses of the behavior of spin-polarized matter [14, frequency standard comparisons [15, 16, 17, 18, Michelson-Morley experiments with cryogenic resonators [19, 20, 21, 22, 23], Doppler effect measurements [24, 25], measurements of neutral meson oscillations [26, 27, 28, 29, 30, 31], polarization measurements on the light from cosmological sources [32, 33, 34, 35], high-energy astrophysical tests [36, 37, 38, 39, 40], precision tests of gravity [41, 42], and others. The results of these experiments set constraints on the various SME coefficients, and up-to-date information about most of these constraints may be found in [43].

Some forms of Lorentz violation - those which break boost invariance - may best be constrained by using relativistic particles. When greater velocities are involved in a test, more stringent bounds on certain SME coefficients are possible. There are two important high-energy processes that are absolutely forbidden in the absence of Lorentz violationvacuum Cerenkov radiation and the photon decay $\gamma \rightarrow e^{+}+e^{-}$. If Lorentz violation appears at high energies, there may be thresholds above which these processes are allowed. The typical scale of such thresholds is $\frac{m}{\sqrt{|\delta|}}$, where $m$ is the mass of the charged particles involved and $|\delta|$ is the dimensionless scale of the Lorentz violation. Consequently, the non-occurrence of these processes up to an energy $E$ will constrain the relevant forms of 
Lorentz violation at the $\mathcal{O}\left(\frac{m^{2}}{E^{2}}\right)$ level.

The fastest-moving particles we can study are astrophysical in origin, and astrophysical observations have been used to place a number of very strong bounds. However, it is also desirable to have laboratory bounds on the SME coefficients. Bounds that are based on energetic astrophysical phenomena may rely on our having an accurate understanding of processes occurring very far away. In many cases, what particle interactions are responsible for a particular experimentally observed phenomenon may only be inferred, not determined directly; and these inferences may be controversial. A relevant example is the disagreement whether $\mathrm{TeV} \gamma$-rays that reach the Earth are produced mostly by inverse Compton scattering or $\pi^{0}$ decay. Conclusions that are based on inferences about astrophysical processes are useful; however, they should also be used with some caution. Such conclusions may not be as reliable as similar ones based on observations made directly in the laboratory.

We shall conclude this introductory section by presenting the SME Lagrangian relevant for interactions among electron, positrons, and photons at high energies. In section 2 we shall discuss the how the velocity, momentum, and other kinematic quantities are related in the Lorentz-violating theory. Section 3 examines how the absence of vacuum Cerenkov radiation from electron and positron beams at LEP, as well as the absence of photon decay, can be used to place bounds on SME parameters. In section 4, we discuss the bounds that can be placed by studying the actual radiation emission (which is synchrotron in origin) from the LEP beams. The paper concludes with section 5, which collects the best reliable bounds that can be derived from all the various laboratory techniques and discusses the outlook for future improvements.

The electron and photon sectors of the minimal SME are described by the Lagrange density

$$
\begin{aligned}
\mathcal{L} & =-\frac{1}{4} F^{\mu \nu} F_{\mu \nu}-\frac{1}{4} k_{F}^{\mu \nu \rho \sigma} F_{\mu \nu} F_{\rho \sigma}+\frac{1}{2} k_{A F}^{\mu} \epsilon_{\mu \nu \rho \sigma} F^{\nu \rho} A^{\sigma}+\bar{\psi}\left(i \Gamma^{\mu} D_{\mu}-M\right) \psi \\
\Gamma^{\mu} & =\gamma^{\mu}+c^{\nu \mu} \gamma_{\nu}-d^{\nu \mu} \gamma_{\nu} \gamma_{5}+e^{\mu}+i f^{\mu} \gamma_{5}+\frac{1}{2} g^{\lambda \nu \mu} \sigma_{\lambda \nu} \\
M & =m+\not \phi-\not \gamma \gamma_{5}+\frac{1}{2} H^{\mu \nu} \sigma_{\mu \nu}+i m_{5} \gamma_{5} .
\end{aligned}
$$

The unconventional behavior of electrons and photons at high energies is primarily determined by the coefficients $c, d$, and $k_{F}$, which are dimensionless and preserve CPT. The fermion coefficients $c$ and $d$ are traceless, while $k_{F}$ has a vanishing double trace and exhibits the same symmetries as the Riemann tensor. These coefficients break rotation, boost, and parity symmetries, and they modify the velocities of electrons, positrons, and photons. However, the usual $e v^{\mu} A_{\mu}$ coupling between charged sources and the electromagnetic field is unchanged; this is a consequence of electromagnetic gauge invariance. Since non-gravitational Lorentz violation is necessarily a small effect, we shall only consider the leading-order effects of the various SME coefficients; at that order, only the symmetric 
parts of $c$ and $d$ can contribute to physical effects.

\section{Lorentz-Violating Kinematics}

The $c, d$, and $k_{F}$ coefficients affect the energy-momentum relations for relativistic quanta. In the electron sector, the changes are most easily expressed in terms of modifications to the maximum achievable velocity (MAV) of a particle. The MAV in the direction $\hat{v}$ is [44]

$$
1+\delta(\hat{v})=1-c_{00}-c_{(0 j)} \hat{v}_{j}-c_{j k} \hat{v}_{j} \hat{v}_{k}+s d_{00}+s d_{(0 j)} \hat{v}_{j}+s d_{j k} \hat{v}_{j} \hat{v}_{k},
$$

where $s$ the product of a particle's helicity and fermion number $(+1$ for electrons, -1 for positrons). The parentheses indicate a symmetrized sum, such as $c_{(0 j)}=c_{0 j}+c_{j 0}$. The dispersion relation for a relativistic particle is $E=\sqrt{m^{2}+p^{2}[1+2 \delta(\hat{p})]}$; this determines the threshold for various novel processes that depend on the existence of Lorentz violation. The velocity at ultrarelativistic energies is

$$
\vec{v}=\left[1+\delta(\hat{p})-\frac{m^{2}}{2 E^{2}}\right] \hat{p}
$$

which is the usual velocity plus $\delta(\hat{p}) \hat{p}$.

The effects in the electromagnetic sector are similar. Photons, being massless, always move with their MAV. The spin-dependent part of $k_{F}$ is extremely tightly constrained experimentally; it gives rise to birefringence that is not seen, even for photons that have traversed cosmological distances. The non-birefringent part is $\tilde{k}^{\mu \nu}=\left(k_{F}\right)_{\alpha}{ }^{\mu \alpha \nu}$, which changes the speed of light in the direction $\hat{v}$ to $1+\delta_{\gamma}(\hat{v})=1-\frac{1}{2}\left[\tilde{k}_{00}+2 \tilde{k}_{0 j} \hat{v}_{j}+\tilde{k}_{j k} \hat{v}_{j} \hat{v}_{k}\right]$. Most observable relativistic effects depend on the difference between the velocity of a moving charge and the speed of light in the same direction. For example, if an electron's speed exceeds $1+\delta_{\gamma}(\hat{v})$, vacuum Cerenkov radiation will be emitted; this is analogous to ordinary Cerenkov emission in matter, where the speed of light in a material is less than 1.

We may choose coordinates in such a way as to eliminate the $c$ or $\tilde{k}$ coefficients in one sector of the theory. (Only differences between these coefficients in different sectors are physically observable.) We shall use this coordinate freedom to make the photon sector conventional. All the constraints we derive will therefore be formulated as limits on combinations of electron coefficients. However, bounds on $c^{\nu \mu}$ should be reinterpreted as bounds on the linear combinations $c^{\nu \mu}-\frac{1}{2} \tilde{k}^{\mu \nu}$ if the electromagnetic $\tilde{k}$ coefficients are not set to zero.

Constraints on SME coefficients are conventionally stated in a Sun-centered celestial equatorial coordinate frame [45]. The origin lies at the center of the Sun. The $Z$-axis points along the Earth's rotation axis; the $X$-axis points toward the vernal equinox point on the celestial sphere; and the $Y$-axis is set by the right hand rule. Time in these coordinates is $T$. 


\section{Vacuum Cerenkov Radiation and Photon Decay}

We are interested in the effects of Lorentz violation on relativistic electron-photon dynamics in terrestrial laboratories. The most energetic Earthbound electron and positron beams were created at the Large Electron-Positron Collider (LEP). In order to study direction-dependent effects, we must understand the range of beam velocities that were possible at this accelerator. In the course of each revolution around a circular accelerator, a particle moves in every direction that lies in the plane of the accelerator ring. As the Earth rotates, this plane rotates as well. If the accelerator is locally horizontal, the plane of the accelerator ring sweeps out the exterior of a double cone. The accelerated particles will travel in every direction $\hat{v}$ that satisfies $|\hat{v} \cdot \hat{Z}| \leq \sin \chi$ (where $\chi$ is the colatitude of the laboratory) during the course of one sidereal day. LEP was located at the European Organization for Nuclear Research (CERN), at latitude $46.2^{\circ} \mathrm{N}$.

It is immediately natural to ask what effect the Lorentz-violating threshold for vacuum Cerenkov radiation would have had on LEP's operation. The threshold for an electron or positron to emit such radiation is [46]

$$
E_{T}=\frac{m}{\sqrt{2 \delta(\hat{v})}} .
$$

This is the energy at which the charge's speed reaches the speed of light. $E_{T}$ is a real energy only if $\delta(\hat{v})>0$; if $\delta(\hat{v})<0$, the MAV is less than 1 .

The instantaneous absence of vacuum Cerenkov radiation indicates that

$$
\delta(\hat{v})<A \equiv \frac{m^{2}}{2 E^{2}}
$$

because the non-radiating particles cannot be moving superluminally. The Cerenkov bound supplies only a one-sided constraint on $c$; the absence of photon decay can supply the other side. However, the vacuum Cerenkov bounds constrain the $d$-dependent part of $\delta(\hat{v})$ from both sides. Both helicity states were represented in the LEP beams, and if Cerenkov radiation from either spin state were allowed, it would have been observed. However, the bounds on $d$ turn out not to be that interesting. All nine of these spindependent coefficients have already been constrained using laboratory experiments with torsion pendulums [14. The pendulum bounds are substantially stronger than the bounds quoted here, although they are not on the $d$ coefficients individually but on particular linear combinations of the $d$ and other parameters. However, it would require remarkable fine tuning for the observable combinations to be as small as they are without the $d$ coefficients also being well below the $10^{-11}-10^{-12}$ scale accessible via accelerators.

Vacuum Cerenkov radiation is an extremely efficient form of energy loss. If the maximum LEP beam energy of $E_{b}=104.5 \mathrm{GeV}$ were only $1 \%$ above the Cerenkov threshold $E_{T}$, the expected time required for a superluminal electron or positron to emit a $\gtrsim 1 \mathrm{GeV}$ photon and drop below the Cerenkov threshold would be $\lesssim 10^{-9}$ s. During this time, the 
particle would advance about $0.2 \mathrm{~m}$ along the $27 \mathrm{~km}$ track, and its direction would not change appreciably. The time required for vacuum Cerenkov emission to drain away a substantial amount of the beam's energy is so short that we may conclude that for every direction along the beam's path, $E_{b}$ is no greater than $1.01 E_{T}$. The right-hand-side of (7) may therefore be taken to be $A=\frac{m^{2}}{2\left(0.99 E_{b}\right)^{2}}=1.2 \times 10^{-11}$ [47].

The survival of energetic photons (through the absence of $\gamma \rightarrow e^{+}+e^{-}$) can also be used to place constraints on Lorentz violation; if this process were allowed, it would operate extremely rapidly [48, 49]. The threshold for photon decay is $2\left|E_{T}\right|$, defined only if $E_{T}$ as given by (6) is imaginary. The absence of this process for photons of energy $E_{\gamma}$ moving in the direction $\hat{v}$ implies

$$
c_{00}+c_{(0 j)} \hat{v}_{j}+c_{j k} \hat{v}_{j} \hat{v}_{k}<B \equiv \frac{2 m^{2}}{E_{\gamma}^{2}} .
$$

The bound (8) does not involve $d$. The reason is that the electron and positron produced by a single photon decaying at threshold have parallel spins. Their energy-momentum relations are shifted in opposite directions by $d$; as a consequence, the presence of $d$ will not make the otherwise forbidden process allowed. This "no-go" conclusion can be evaded by involving additional fields in the decay or taking into account angular momentum nonconservation, which is second order in the Lorentz violation; however, these cannot induce the kind of rapid photon decay that may be seen with nonzero $c$.

Collision events at accelerators produce many photons. Photons were produced at the Tevatron with energies above $300 \mathrm{GeV}$ [50]. However, matters are complicated by the fact that the range of photon directions is essentially the entire sphere. If isotropy is assumed, then the observed survival of these photons may easily be converted into bounds on the SME coefficient $c_{00}$; However, if anisotropy is allowed, the analysis of the accelerator-produced photons becomes much more complicated. Moreover, even with the simplification of assumed rotation invariance, these data do not provide a particularly efficient way to set reliable constraints on Lorentz violation. For one thing, the laboratory origin of the photons is not a requirement to produce a reliable bound. Any photon, regardless of origin, that is observed to have propagated a substantial distance is a demonstration that $\gamma \rightarrow e^{+}+e^{-}$is not allowed at the momentum involved. The detection of the photon, which is the only part of its history that really matters, is just as much a reliable, laboratory measurement as the observation of circulating beams at an accelerator. Cosmic ray $\gamma$-rays have been observed with energies of nearly $100 \mathrm{TeV}$, which should lead to stronger bounds than would be possible with accelerator data alone.

We shall therefore not discuss the decay of photons produced at accelerators any further, except to note the following. The beam directions $\hat{v}$ explored at LEP are a subset of the photon directions observed at the Tevatron. If the bounds (77) and (8) were simultaneously satisfied for every direction with $|\hat{v} \cdot \hat{Z}| \leq \sin \chi_{C}$ (where $\chi_{C} \approx 43.8^{\circ}$ is the colatitude at CERN), then for any such direction, we would have the vacuum Cerenkov bounds $c_{T T} \pm c_{(T J)} \hat{v}_{J}+c_{J K} \hat{v}_{J} \hat{v}_{K}-\left|d_{T T} \pm d_{(T J)} \hat{v}_{J}+d_{J K} \hat{v}_{J} \hat{v}_{K}\right|>-A$ and photon decay 
bounds $c_{T T} \pm c_{(T J)} \hat{v}_{J}+c_{J K} \hat{v}_{J} \hat{v}_{K}<B$. (The \pm arises from taking into account the motion both along $\hat{v}$ and $-\hat{v}$.) It would then be straightforward to separate the $c$ and $d$ bounds and to further disentangle the even- and odd-parity coefficients. Adding and subtracting these inequalities yields

$$
\begin{aligned}
-A<c_{T T}+c_{J K} \hat{v}_{J} \hat{v}_{K} & <B \\
\left|c_{(T J)} \hat{v}_{J}\right| & <(A+B) / 2 \\
\left|d_{T T}+d_{J K} \hat{v}_{J} \hat{v}_{K}\right| & <A+B \\
\left|d_{(T J)} \hat{v}_{J}\right| & <A+B .
\end{aligned}
$$

These would be the maximal anisotropic generalizations of the LEP vacuum Cerenkov radiation and Tevatron photon decay results of [47]. They would be sufficient to establish completely separate bounds on the six $c_{(T J)}$ and $d_{(T J)}$ coefficients, by choosing $\hat{v}=\hat{X}$, $\hat{v}=\hat{Y}$ and $\hat{v}=\hat{X} \cos \chi_{C} \pm \hat{Z} \sin \chi_{C}$. [The resulting bound on, for example, $c_{(T Z)}$ would be $\left|c_{(T Z)}\right|<\frac{A+B}{2 \sin \chi_{C}}$.] However, the other inequalities (9) and (11) could not be further disentangled using only this data. We shall therefore turn our attention to other processes that can occur at accelerators.

\section{Synchrotron Radiation}

Vacuum Cerenkov radiation and photon decay are normally forbidden, but they may occur in the presence of Lorentz violation. On the other hand, the most important electron-photon interaction that we know really does occur at particle accelerators is synchrotron radiation, and information about real synchrotron losses can provide further constraints on electron Lorentz violation. Information about these losses provides the strongest two-sided laboratory bounds on $c_{T T}$, and the radiation spectrum is sensitive to rotation noninvariance coefficients as well. Synchrotron motion and radiation in the presence of $c$ was discussed in [51]. The basic picture is fairly simple, and the instantaneous radiation rate depends on the difference between an orbiting charge's speed and the speed of light in the direction the charge is moving.

With $k_{F}=0$, the electromagnetic sector behaves according to ordinary special relativity; the electromagnetic field responds to charged particles normally, and the radiation emitted is determined in the usual way by the velocity profiles of the moving charges. The

total radiated synchrotron power is $P=\frac{e^{2} a^{2}}{6 \pi m^{2}} \gamma^{4}$, where $a$ is the magnitude of the magnetic acceleration. [If photon-sector Lorentz violation were included, the electromagnetic sector would behave (in the narrow pencil of angles into which the synchrotron radiation is beamed) like standard special relativity, except with a modified Lorentz factor

$$
\tilde{\gamma}=\frac{1}{\sqrt{1+2 \delta_{\gamma}(\hat{v})-v^{2}}} .
$$


The power radiated would then be $P=\frac{e^{2} a^{2}}{6 \pi m^{2}} \tilde{\gamma}^{4}$.] For ultrarelativistic particles, $\gamma \approx$ $[2(1-v)]^{-1 / 2}$ increases very rapidly as a function of $v$, since $\frac{d \gamma}{d v}=v \gamma^{3} \approx \gamma^{3}$. The modified expression for $\vec{v}(\vec{p})$ changes the radiated power $P(\vec{p})$ to

$$
P(\vec{p})=P_{0}(\vec{p})\left\{1+4 \gamma^{2}\left[\delta(\hat{p})-\delta_{\gamma}(\hat{p})\right]\right\},
$$

where $P_{0}(\vec{p})$ is the radiation rate for a particle of momentum $\vec{p}$ in the absence of any Lorentz violation. The fractional change in the radiated power increases with the square of the energy.

The beam energy at LEP was determined very precisely, because making high-precision measurements of the $\mathrm{W}$ and $\mathrm{Z}$ boson masses was one of the accelerator's most important functions. The beam energy $E_{b}$ was calculated using several different methods, which could be used to double-check one-another. The primary method was based on measuring the magnetic field profile along the beam's trajectory (which was also carefully measured). The field measurements used nuclear magnetic resonance (NMR) probes. The validity of the NMR measurements should not be affected by Lorentz violation; Lorentz violation strong enough to affect the NMR is already ruled out by atomic clock experiments [15, [16, 17, 18. The field profile and the trajectory through the bending magnets were known to high precision, and together these determined $E_{b}$.

A complementary measurement used the synchrotron tune, $Q_{s}$ - the ratio of the synchrotron oscillation frequency to the orbital frequency [52]. Synchrotron oscillations occur because of nonuniformity in beam particles' energies. Particles with less than the nominal beam energy curve more sharply and thus travel around smaller orbits. This means they take less time to travel between the accelerating radio frequency ( $\mathrm{RF}$ ) cavities. The less energetic particles therefore arrive at the cavities earlier in the $\mathrm{RF}$ cycle and receive larger-than-expected energy boosts, moving their energy back toward the nominal beam energy. An opposite effect occurs for particle with greater than the nominal energy. These effects cause oscillations in the energy of the beam, and a fit to their frequency provides an independent way to determine the central energy $E_{b}$. The best measurement of the synchrotron tune at LEP came from runs at $E_{b}=91 \mathrm{GeV}$, near the $\mathrm{Z}$ pole. The Lorentz factor at this energy is $\gamma>1.7 \times 10^{5}$. Fitting the synchrotron oscillations at this value of the beam energy, the difference between the values of the energy inferred from the tune $Q_{s}$ and from the magnetic field profile was only $3 \mathrm{MeV}$; this discrepancy was small compared with the $1 \sigma$ uncertainty of $21 \mathrm{MeV}$ that was associated with the fitting procedure and with the imprecision in the actual measurement of $Q_{s}$.

The formula for $Q_{s}$ depends on $E_{b}$ and the energy loss per orbit $U_{0}$, in the combination $\left(g^{2} e^{2} V_{R F}^{2}-U_{0}^{2}\right) / E_{b}^{2} . V_{R F}$ is the amplitude of the RF voltage during the beam's passage through the accelerating cavities; $\psi_{s}$ is the phase of the accelerating voltage, and under the steady state conditions at which the collider operated, $U_{0}=g e V_{R F} \sin \psi_{s}$. Both $V_{R F}$ and $\psi_{s}$ are known to high precision. The factor $g$ is a correction associated with possible phasing errors and misalignments of the RF cavities; $g$ must be determined as part of the fit for $Q_{s}$, although its value is necessarily close to 1 . The fitting required makes $g$ 
a significant source of uncertainty. However, the fitting can be done on data taken at well below the usual experimental energies, since the magnet alignment and RF cavity timing do not depend on the operating energy. At low energies, $g$ can be determined more precisely than would be possible at higher energies; and the fitted value of the correction factor can then be carried over to the actual $91 \mathrm{GeV}$ running energy. The possible presence of Lorentz violation changes the formula for $Q_{s}$ only through a rescaling of $U_{0}$; the Lorentz force law is not modified, and the energy imparted by the RF cavities is unchanged.

In the fit used to determine $Q_{s}$, it was assumed that $U_{0}$ had the usual synchrotron form plus small corrections. The corrections are necessary to account for other forms of energy loss, primarily through parasitic impedance interactions between the beam and the apparatus or effects associated with finite beam size. However, these corrections could be either modeled from first principles or measured directly. Since the NMR measurements provided an independent measure of the beam energy $E_{b}$, it is possible to reinterpret the fit for $Q_{s}$ as a measurement not of $E_{b}$ but rather of $U_{0}$. Because $E_{b}$ and $U_{0}$ enter the formula for $Q_{s}$ primarily in the combination $\left(U_{0} / E_{b}\right)^{2} \cos ^{2} \psi_{s}$, if the fit (assuming a known form for $U_{0}$ ) could determine $E_{b}$ with a fractional uncertainty $\eta$, the same fit would (if $E_{b}$ were instead taken to be known) determine $U_{0}$ with the same fractional precision. Since $U_{0}$ is determined by the synchrotron radiation power $P$, this immediately places a bound on the SME contribution to $P$ as given in (14):

$$
|\langle\delta\rangle|<C \equiv \eta \frac{m^{2}}{4 E_{b}^{2}},
$$

where $\langle\delta\rangle$ denotes the average value of $\delta(\hat{p})$ over one orbit around the accelerator.

In $\langle\delta\rangle$, the parity-odd $c_{0 j}$ coefficients average to zero. The effects of these coefficients on the speed $v$ change sign when the momentum is reversed; they cannot contribute to the average radiation emitted over a full revolution, because orbiting particles experience equal and opposite velocities at antipodal points along their orbits. The effects of the $d$ coefficients are also averaged out. The $d$ coefficients would affect the speed and hence the synchrotron losses if the beams were consistently longitudinally polarized. However, such polarization cannot be maintained over long periods; helicities evolve in the magnetic field, because of particles' anomalous magnetic moments. The beams at LEP were maintained through most of their orbits in transverse polarization states; if desired, they could be temporarily rotated into longitudinal polarization states immediately before an interaction point. The average value of $\delta(\hat{p})$ is thus $-c_{00}-\frac{1}{2} c_{j} c_{k}\left[\left(\hat{e}_{1}\right)_{j}\left(\hat{e}_{1}\right)_{k}+\left(\hat{e}_{2}\right)_{j}\left(\hat{e}_{2}\right)_{k}\right]$, where $\hat{e}_{1}$ and $\hat{e}_{2}$ are perpendicular unit vectors in the plane of the orbit; a particle essentially performs half its motion in the $\pm \hat{e}_{1}$-direction and half in the $\pm \hat{e}_{2}$-direction. Using the tracelessness of $c^{\nu \mu}$, the average may be more concisely written as

$$
\langle\delta\rangle=-\frac{3}{2} c_{00}+\frac{1}{2} c_{j k} \hat{n}_{j} \hat{n}_{k},
$$


where $\hat{n}$ is the unit vector normal to the circular path.

The fractional uncertainty assigned to the value of $E_{b}$ that was derived from fitting the synchrotron tune was $\eta=2.4 \times 10^{-4}$. This could then be interpreted as the fractional error in $U_{0}$ and hence $P$. A somewhat more conservative estimate of the possible error is $\eta<6 \times 10^{-4}$; this is a $2 \sigma$ error, and it also accounts for other sources of error, such as in the NMR measurement of $E_{b}$ and the discrepancy between the NMR and $Q_{s}$ measurements of the energy. Using this value for $\eta$, we have $C=5 \times 10^{-15}$.

This provides a bound on a combination of boost and rotation invariance violation coefficients. The relevant $\hat{n}$ appearing in (16) is the normal vector to the orbit at the time when the synchrotron tune data used in the energy calibration was collected. If the tune data was collected over an extended period (covering many values of $\hat{n}$ ), and $Q_{s}$ was consistent across all these measurements, the bound (15) would hold for all the directions $\hat{n}$ that the accelerator passed through - all $\hat{n}$ with $\hat{n} \cdot \hat{Z}=\cos \chi_{C}$. However, this conclusion does not appear to be supported; it is only the average value of the synchrotron tune that was used for the energy fitting, and we cannot rule out that there were small sidereal variations in $Q_{s}$. We could use the sidereally averaged value $\left\langle c_{j k} \hat{n}_{j} \hat{n}_{k}\right\rangle=\frac{1}{2} \sin ^{2} \chi_{C}\left(c_{X X}+\right.$ $\left.c_{Y Y}\right)+\cos ^{2} \chi_{C} c_{Z Z}$, but there are other, stronger laboratory bounds on the $c_{J K}$ coefficients, which will described in section 5, and the existence of these $c_{J K}$ bounds makes (15) important primarily as a bound on $c_{T T}$.

\section{Best Combined Bounds on $c^{\nu \mu}$}

We shall finally turn our attention to how the high-energy bounds (and other, low-energy bounds to be described shortly) can be merged together to produce the best laboratory constraints on the $c^{\nu \mu}$ coefficients. We shall pay particular attention to those coefficients, $c_{T T}$ and $c_{(T J)}$ associated with the breaking of boost invariance. (The $d^{\nu \mu}$ coefficients, as previously noted, are already extremely well constrained by nonrelativistic spin measurements.)

The synchrotron bounds take the form $\left|\frac{3}{2} c_{T T}-\frac{1}{2} c_{J K} \hat{n}_{J} \hat{n}_{K}\right|<C$. Regardless of what direction $\hat{n}$ is used, this implies that $\left|c_{T T}\right|<\frac{3}{4}(C+\lambda)$, where $\lambda$ is the largest singular value of the traceless $3 \times 3$ matrix

$$
c_{J K}-\frac{1}{3} c_{T T} \delta_{J K}=\frac{1}{2}\left[\begin{array}{ccc}
c_{-}+\frac{1}{3} c_{Q} & c_{(X Y)} & c_{(X Z)} \\
c_{(X Y)} & -c_{-}+\frac{1}{3} c_{Q} & c_{(Y Z)} \\
c_{(X Z)} & c_{(Y Z)} & -\frac{2}{3} c_{Q}
\end{array}\right] .
$$

The quantities $c_{-}=c_{X X}-c_{Y Y}, c_{Q}=c_{X X}+c_{Y Y}-2 c_{Z Z}$, and $c_{(J K)}$ are those that can be bounded in stationary tests of isotropy. The singular value must satisfy

$$
\lambda \leq \frac{1}{\sqrt{2}}\left[\left|c_{(X Y)}\right|^{2}+\left|c_{(X Z)}\right|^{2}+\left|c_{(Y Z)}\right|^{2}+\left|c_{-}\right|^{2}+\frac{1}{3}\left|c_{Q}\right|^{2}\right]^{1 / 2} .
$$


The existence of multiple complementary bounds on the types of Lorentz violation [20, 21, 22 relevant to optical cavity experiments should imply bounds on the $c$ coefficients appearing in (18), each at the $10^{-17}$ level. The key point in the derivation of such electron bounds from Michelson-Morley-type measurements is the use of optical cavities containing different materials. The dimensions of a block of material determine its resonant frequencies when it is used as an optical cavity. However, these dimensions are determined by the material's electron structure and are affected by electron Lorentz violation. This makes the Michelson-Morley experiments sensitive to both photon and electron sector SME coefficients. Cavity materials with different electronic properties are sensitive to different mixtures of electron and photon parameters, so with multiple experiments, it is possible to disentangle the bounds from the two sectors 23 .

An up-to-date analysis of these bounds has not been performed. Older resonator data provided bounds on all the $c$ coefficients appearing in (18) at the $10^{-16}$ level, except for $c_{Q}$, which was bounded at only the $10^{-14}$ level [19]. However, we shall assume that an improved analysis based on [20, 21, 22] will indeed produce bounds on the five anisotropic $c_{J K}$ coefficients (and hence $\lambda$ ) at approximately the $10^{-17}$ level. This means that these coefficients can effectively be neglected in the analysis of the accelerator results, for purposes of determining the strongest laboratory bounds that are available on $c_{T T} ; \lambda$ is very small compared to $C$. This implies that

$$
\left|c_{T T}\right|<4 \times 10^{-15}
$$

this is the same bound given in [53], but expressed in different notation, with the photon sector Lorentz violation set to zero.

The synchrotron bound on $c_{T T}$, the resonant cavity bounds on the anisotropic $c_{J K}$, the torsion pendulum bounds on the $d^{\nu \mu}$, and the vacuum Cerenkov bounds (7) can be further combined to establish new, accelerator-based constraints on the $c_{(T J)}$ parameters. Because of the smallness of the $\left|c_{J K}\right| \lesssim \lambda$ and $\left|c_{T T}\right| \lesssim C$, in conjunction with the hierarchy $\lambda \ll C \ll A$, the $\delta(\hat{v})$ appearing on the left-hand side of (7) will be dominated by $\pm v_{J} c_{(T J)}$ if the Lorentz violation is at the greatest allowed level. The corresponding bounds are (neglecting $d$ entirely) $\left|v_{J} c_{(T J)}\right|<A+C+2 \lambda$, or

$$
\begin{aligned}
\left|c_{(T X)}\right|,\left|c_{(T Y)}\right| & <1.2 \times 10^{-11} \\
\left|c_{(T Z)}\right| & <1.8 \times 10^{-11} .
\end{aligned}
$$

These are an order of magnitude better than the best atomic clock bounds on these kinds of parameters [54.

However, since the reliability of a bound based on the absence of $\gamma \rightarrow e^{+}+e^{-}$does not depend on how the $\gamma$-ray involved originated, we can improve these bounds using astrophysical observations. In [55], we discussed the bounds that could be placed on $c$ using astrophysical photon survival data and compiled a list of sources that produce $\gamma$-rays with $E_{\gamma} / m_{e}$ in the $10^{7}-10^{8}$ range. To each such source, there corresponds a constraint of 


\begin{tabular}{|l|c|c|c|c|}
\hline Emission source & $\hat{v}_{X}$ & $\hat{v}_{Y}$ & $\hat{v}_{Z}$ & $E_{\gamma} / m_{e}$ \\
\hline Cas A & -0.51 & 0.08 & -0.86 & $8 \times 10^{6}[56]$ \\
Crab nebula & -0.10 & -0.92 & -0.37 & $1.6 \times 10^{8}[57]$ \\
G 12.82-0.02 & -0.06 & 0.95 & 0.29 & $5 \times 10^{7}[58]$ \\
G 18.0-0.7 & -0.11 & 0.97 & 0.24 & $7 \times 10^{7}[59,60]$ \\
G 106.3+2.7 & -0.45 & 0.19 & -0.87 & $2 \times 10^{7}[61]$ \\
G 347.3-0.5 & 0.16 & 0.75 & 0.64 & $10^{8}[62]$ \\
J1427-608 & 0.39 & 0.29 & 0.87 & $6 \times 10^{7}[63]$ \\
J1626-490 & 0.26 & 0.60 & 0.75 & $6 \times 10^{7}[63]$ \\
J1731-437 & 0.09 & 0.72 & 0.69 & $6 \times 10^{7}[63]$ \\
J1745-303 & 0.06 & 0.86 & 0.50 & $4 \times 10^{7}[64]$ \\
J1841-055 & -0.18 & 0.98 & 0.10 & $4 \times 10^{7}[63]$ \\
J1857+026 & -0.25 & 0.97 & -0.05 & $8 \times 10^{7}[63]$ \\
M87 & 0.97 & 0.13 & -0.21 & $2 \times 10^{7}[65]$ \\
Mkn 421 & 0.76 & -0.19 & -0.62 & $3 \times 10^{7}[66,67]$ \\
Mkn 501 & 0.22 & 0.74 & -0.64 & $4 \times 10^{7}[68]$ \\
MSH 15-52 & 0.34 & 0.38 & 0.86 & $8 \times 10^{7}[69]$ \\
Vela SNR & 0.44 & -0.55 & 0.71 & $1.3 \times 10^{8}[70]$ \\
\hline
\end{tabular}

Table 1: Energies of observed $\gamma$-rays from various astrophysical sources. References are given for each value of the energy.

the form (8), parameterized by the direction $\hat{v}$ from the source to the Earth. In terms of the right ascension $\alpha$ and declination $\delta$, the components of this $\hat{v}$ are $\hat{v}_{X}=-\cos \delta \cos \alpha$, $\hat{v}_{Y}=-\cos \delta \sin \alpha$, and $\hat{v}_{Z}=-\sin \delta$. Using only the photon survival data, it was not possible to place two-sided constraints. However, two-sided bounds on the $c_{(T J)}$ are again possible if the photon survival data is combined with the same laboratory bounds on $c_{T T}$ and $c_{J K}$ that were discussed in the preceding paragraph.

Table 1 gives an updated list of sources for which TeV photon observations have been made. (This list is not exhaustive. There are other sources from which comparably energetic photons have been observed, but they are not included if the do not affect the output of the linear program discussed below. A source may be superfluous in this way if, for example, it is located quite close to another source, so that the two sources produce bounds on very similar linear combinations of coefficients.) Most of the best measurements of ultra-high-energy photon spectra have been made by the H.E.S.S. atmospheric Cerenkov telescope in Namibia and the newer VERITAS telescope in Arizona. These devices are extremely sensitive, but they have limited sky coverage.

To separate the $c_{(T J)}$ bounds from those on other coefficients requires linear programming, but the results are straightforward. Using the the data from table 1 in the inequality (8), as well as using the inequality (19) [inclusion of the LEP photon survival 


\begin{tabular}{|c|c|c|}
\hline$c^{\mu \nu}$ & Maximum & Minimum \\
\hline$c_{T T}$ & $2 \times 10^{-15}$ & $-4 \times 10^{-15}$ \\
$c_{(T X)}$ & $10^{-14}$ & $-3 \times 10^{-13}$ \\
$c_{(T Y)}$ & $6 \times 10^{-15}$ & $-8 \times 10^{-14}$ \\
$c_{(T Z)}$ & $1.3 \times 10^{-13}$ & $-1.1 \times 10^{-14}$ \\
\hline
\end{tabular}

Table 2: Disentangled bounds on the boost invariance violation components of $c$.

results, in the form of inequalities (20) and (21), turns out to be unnecessary], we have extracted bounds on all the boost-invariance-violating $c$ coefficients. Because the resulting bounds are significantly less stringent than the expected Michelson-Morley bounds on the $c_{J K}$, we can neglect the $c_{J K}$ entirely in our analysis.

The results from the linear program are given in table2. The values listed in the table are the absolute maximum and minimum values of the $c_{T T}$ and $c_{(T J)}$ coefficients that are consistent with the data. No restrictions on any other coefficients are assumed, except with regard to a convention for choosing coordinates. Our convention has been to set $\tilde{k}^{\mu \nu}=0$. If the coordinates are not chosen so as to make the photon sector conventional, all these bounds are really on linear combinations $c^{\nu \mu}-\frac{1}{2} \tilde{k}^{\mu \nu}$. In terms of the $\tilde{\kappa}$ three-tensors into which $k_{F}$ is usually decomposed, the bounds are on the combinations $c_{T T}-\frac{3}{4} \tilde{\kappa}_{\mathrm{tr}}$ and $c_{(T J)}-\frac{1}{2} \epsilon_{J K L}\left(\tilde{\kappa}_{O+}\right)_{K L}$. None of the bounds in table 2 are as strong as the bounds on the same quantities from [39], which were derived by assuming we possess an accurate understanding of how the sources involved are producing their spectra. However, for the upper bound on $c_{(T Y)}$, the difference in precision is less than an order of magnitude.

Which physical phenomena are responsible for which of the bounds deserves some explanation. The lower bound on $c_{T T}$ is simply that of (19), but the upper bound also depends on both H.E.S.S. and VERITAS astrophysical photon survival data. The upper bounds on $c_{(T X)}$ and $c_{(T Y)}$ and the lower bound on $c_{(T Z)}$ come from combining the H.E.S.S. data with the synchrotron bounds on $c_{T T}$. The remaining three bounds - the lower bounds on $c_{(T X)}$ and $c_{(T Y)}$ and the upper bound on $c_{(T Z)}$-are roughly an order of magnitude weaker. These bounds depend on the inclusion of VERITAS data, which are not yet as comprehensive as the H.E.S.S. data at the very highest energies. Yet without the the VERITAS observations, the bounds in question would be at the $10^{-11}$ level corresponding to (20) and (21).

It is also important to understand the advantages and limitations of each of the methods of bounding $c_{T T}$ and $c_{(T J)}$. The strength of the $c_{T T}$ bounds is a consequence of the quality of the LEP energy calibration data. The analysis of the of the synchrotron spectrum gives bounds that are proportional to $\frac{m^{2}}{E^{2}}$; the same factor determines the strength of the threshold bounds. However, the bounds involving thresholds for new processes cannot be improved by more precise measurements; if a decay process like vacuum Cerenkov radiation is allowed at a certain energy, it will generally occur quite quickly and be easily 
detectable. If no such decays are seen to occur, a bound on the SME coefficients immediately follows. The difference with synchrotron radiation is that it occurs whether the theory is conventional or Lorentz violating. This means the spectrum can be measured precisely, and the precision factor $\eta$ improves the bounds on the $c$ coefficients involved. In principle, the instantaneous synchrotron spectrum is sensitive to all the electron $c$ coefficients. However, the total emission over one orbit is the only quantity that has been accurately determined, and this is sensitive only to a particular combination of coefficients - $\langle\delta\rangle$ from (15) - which does not involve the $c_{(T J)}$.

The observed absences of $e^{ \pm} \rightarrow e^{ \pm}+\gamma$ and $\gamma \rightarrow e^{+}+e^{-}$up to an energy $E$ also constrain $c_{T T}$ and $c_{(T J)}$ at the $\mathcal{O}\left(\frac{m^{2}}{E^{2}}\right)$ level. The LEP data show the absence of vacuum Cerenkov radiation up to beam energies of $104.5 \mathrm{GeV}$. This is a much lower energy than can be seen in cosmic ray photons; the raw bounds (8) derived from the H.E.S.S. observations are $4-5$ orders of magnitude stronger than the LEP bounds (7). However, the advantage of the accelerator data is that it is available and consistent over quite a broad range of directions $\hat{v}$. Until the publication of the VERITAS data, the H.E.S.S. data alone were insufficient to constrain all the $c_{(T J)}$ coefficients from above and below; the LEP results would have been needed to fill in some gaps.

Now, with VERITAS accumulating data and covering a quite different area of the sky from H.E.S.S., those LEP results are no longer needed in order to set reliable laboratory bounds on the $c_{(T J)}$. Naturally, as both $\gamma$-ray telescopes explore more sources for longer times, the quality of the bounds will improve somewhat. It should be possible to improve the laboratory bounds on all the $c_{(T J)}$ to the $10^{-14}$ level or better.

Of course, in deriving the bounds on $c_{T T}$ and $c_{(T J)}$, we have assumed that the Michelson-Morley experiments do indeed place strong enough bounds on all the stationary anisotropy coefficients so that $\lambda$ may effectively be neglected. Existing analyses have not quite achieved this, although the raw Michelson-Morley data certainly appear to be sufficiently precise. Moreover, of the five coefficients that can, according to (18), contribute to $\lambda$, only $c_{Q}$ is potentially large enough to interfere with the results in this paper. It would be quite desirable to have updated bounds on the $c_{J K}$ coefficients, based on the latest generation of resonant cavity experiments. Such bounds would be interesting, both for their influence on the effects discussed here and in their own right. However, if improved bounds on $c_{Q}$ do not actually materialize, the effect on the results of this paper would be modest. None of the bounds in table 2 would be worsened by more than $4 \times 10^{-14}$.

We have discussed several different phenomena that can be observed in the laboratory and used to constrain electron Lorentz violation. However, not all of these methods produce competitive bounds. The best present laboratory techniques for bounding the various SME coefficients we have discussed are: torsion pendulum experiments with spin-polarized samples for $d^{\nu \mu}$, Michelson-Morley experiments with resonators of different compositions for $c_{J K}$, synchrotron radiation at LEP for $c_{T T}$, and observations of photon survival for $c_{(T J)}$. The last of these may involve $\gamma$-rays of astrophysical origin, but which 
are measured in a laboratory on Earth. Using all the observations of such $\gamma$-rays, we have placed new laboratory bounds on all the $c_{(T J)}$ coefficients, at the $10^{-13}-10^{-15}$ levels. [Using more restricted collections of data sets would lead to results such as (9 12) or (20 21).] Some of these bounds are naturally expected to improve as better observational data become available.

\section{References}

[1] D. Colladay, V. A. Kostelecký, Phys. Rev. D 55, 6760 (1997).

[2] D. Colladay, V. A. Kostelecký, Phys. Rev. D 58, 116002 (1998).

[3] V. A. Kostelecký, Phys. Rev. D, 69105009 (2004).

[4] V. A. Kostelecký, C. D. Lane, A. G. M. Pickering, Phys. Rev. D 65, 056006 (2002).

[5] D. Colladay, P. McDonald, Phys. Rev. D 75, 105002 (2007).

[6] V. A. Kostelecký, R. Lehnert, Phys. Rev. D 63, 065008 (2001).

[7] R. Bluhm, V. A. Kostelecký, N. Russell, Phys. Rev. Lett. 79, 1432 (1997).

[8] G. Gabrielse, A. Khabbaz, D. S. Hall, C. Heimann, H. Kalinowsky, W. Jhe, Phys. Rev. Lett. 82, 3198 (1999).

[9] H. Dehmelt, R. Mittleman, R. S. Van Dyck, Jr., P. Schwinberg, Phys. Rev. Lett. 83, 4694 (1999).

[10] R. Bluhm, V. A. Kostelecký, N. Russell , Phys. Rev. Lett. 82, 2254 (1999).

[11] D. F. Phillips, M. A. Humphrey, E. M. Mattison, R. E. Stoner, R. F. C. Vessot, R. L. Walsworth , Phys. Rev. D 63, 111101 (R) (2001).

[12] R. Bluhm, V. A. Kostelecký, C. D. Lane, Phys. Rev. Lett. 84, 1098 (2000).

[13] V. W. Hughes, et al., Phys. Rev. Lett. 87, 111804 (2001).

[14] B. R. Heckel, E. G. Adelberger, C. E. Cramer, T. S. Cook, S. Schlamminger, U. Schmidt, Phys. Rev. D 78, 092006 (2008).

[15] C. J. Berglund, L. R. Hunter, D. Krause, Jr., E. O. Prigge, M. S. Ronfeldt, S. K. Lamoreaux, Phys. Rev. Lett. 75, 1879 (1995).

[16] V. A. Kostelecký, C. D. Lane, Phys. Rev. D 60, 116010 (1999). 
[17] D. Bear, R. E. Stoner, R. L. Walsworth, V. A. Kostelecký, C. D. Lane, Phys. Rev. Lett. 85, 5038 (2000).

[18] P. Wolf, F. Chapelet, S. Bize, A. Clairon, Phys. Rev. Lett. 96, 060801 (2006).

[19] H. Müller, et al., Phys. Rev. Lett. 99, 050401 (2007).

[20] S. Herrmann, A. Senger, K. Möhle, E. V. Kovalchuk, A. Peters, in CPT and Lorentz Symmetry IV, edited by V. A. Kostelecký (World Scientific, Singapore, 2008), p. 9.

[21] S. Herrmann, et al., Phys. Rev. D 80, 105011 (2009).

[22] Ch. Eisele, A. Yu. Nevsky, S. Schiller, Phys. Rev. Lett. 103, 090401 (2009).

[23] H. Müller, Phys. Rev. D 71, 045004 (2005).

[24] G. Saathoff, S. Karpuk, U. Eisenbarth, G. Huber, S. Krohn, R. Muñoz Horta, S. Reinhardt, D. Schwalm, A. Wolf, G. Gwinner, Phys. Rev. Lett. 91, 190403 (2003).

[25] C. D. Lane, Phys. Rev. D 72, 016005 (2005).

[26] V. A. Kostelecký, Phys. Rev. Lett. 80, 1818 (1998).

[27] V. A. Kostelecký, Phys. Rev. D 61, 016002 (1999).

[28] Y. B. Hsiung, Nucl. Phys. Proc. Suppl. 86, 312 (2000).

[29] K. Abe et al., Phys. Rev. Lett. 86, 3228 (2001).

[30] J. M. Link et al., Phys. Lett. B 556, 7 (2003).

[31] B. Aubert et al., Phys. Rev. Lett. 96, 251802 (2006).

[32] S. M. Carroll, G. B. Field, Phys. Rev. Lett. 79, 2394 (1997).

[33] V. A. Kostelecký, M. Mewes, Phys. Rev. Lett. 87, 251304 (2001).

[34] V. A. Kostelecký, M. Mewes, Phys. Rev. Lett. 97, 140401 (2006).

[35] V. A. Kostelecký, M. Mewes, Phys. Rev. Lett. 99, 011601 (2007).

[36] F. W. Stecker, S. L. Glashow, Astropart. Phys. 16, 97 (2001).

[37] T. Jacobson, S. Liberati, D. Mattingly, Nature 424, 1019 (2003).

[38] B. Altschul, Phys. Rev. Lett. 96, 201101 (2006).

[39] B. Altschul, Phys. Rev. D 74, 083003 (2006). 
[40] F. R. Klinkhamer, M. Risse, Phys. Rev. D 77, 016002 (2008); addendum Phys. Rev. D 77, 117901 (2008).

[41] J. B. R. Battat, J. F. Chandler, C. W. Stubbs, Phys. Rev. Lett. 99, 241103 (2007).

[42] H. Müller, S. Chiow, S. Herrmann, S. Chu, Phys. Rev. Lett. 100, 031101 (2008).

[43] V. A. Kostelecký, N. Russell, arXiv:0801.0287.

[44] B. Altschul, D. Colladay, Phys. Rev. D 71, 125015 (2005).

[45] R. Bluhm, V. A. Kostelecký, C. D. Lane, N. Russell, Phys. Rev. D 68, 125008 (2003).

[46] B. Altschul, Phys. Rev. Lett. 98, 041603 (2007).

[47] M. A. Hohensee, R. Lehnert, D. F. Phillips, R. L. Walsworth, Phys. Rev. Lett. 102, 170402 (2009).

[48] S. Coleman, S. L. Glashow, Phys. Rev. D 59, 116008 (1999).

[49] M. A. Hohensee, R. Lehnert, D. F. Phillips, R. L. Walsworth, Phys. Rev. D 80, 036010 (2009).

[50] V. M. Abazov, et al., Phys. Lett. B 639, 151 (2006).

[51] B. Altschul, Phys. Rev. D 72, 085003 (2005).

[52] R. Assmann, et al., Eur. Phys. J. C 39, 253 (2005).

[53] B. Altschul, Phys. Rev. D 80, 091901(R) (2009).

[54] B. Altschul, Phys. Rev. D 81, 041701(R) (2010).

[55] B. Altschul, Astropart. Phys. 28, 380 (2007).

[56] V. A. Acciari, et al., Astrophys. J. 714, 163 (2010).

[57] T. Tanimori, et al., Astrophys. J. Lett. 492, L33 (1998).

[58] F. A. Aharonian, et al., Astrophys. J. 636, 777 (2006).

[59] F. A. Aharonian, et al., Astron. Astrophys. 442, L25 (2005).

[60] F. A. Aharonian, et al., astro-ph/0607548.

[61] V. A. Acciari, et al., Astrophys. J. Lett. 703, L6 (2009).

[62] F. A. Aharonian, et al., Astron. Astrophys. 464, 235 (2007). 
[63] F. A. Aharonian, et al., Astron. Astrophys. 477, 353 (2008).

[64] F. A. Aharonian, et al., Astron. Astrophys. 483, 509 (2008).

[65] F. A. Aharonian, et al., Science 314, 1424 (2006).

[66] J. Albert, et al., Astrophys. J. 663, 125 (2007).

[67] F. A. Aharonian, et al., Astron. Astrophys. 437, 95 (2005).

[68] F. A. Aharonian, et al., Astron. Astrophys. 349, 11 (1999).

[69] F. A. Aharonian, et al., Astron. Astrophys. 435, L17 (2005).

[70] F. A. Aharonian, et al., Astron. Astrophys. 448, L43 (2006). 